Lecture Notes in Mechanical Engineering

Américo Azevedo Editor

Advances in

Sustainable

and Competitive

Manufacturing Systems

23rd International Conference on

Flexible Automation and Intelligent

Manufacturing 


\title{
Modeling and Simulation of the EMG30 Geared Motor with Encoder Resorting to SimTwo: The Official Robot@ Factory Simulator
}

\author{
José Gonçalves, José Lima, Paulo J. Costa and A. Paulo Moreira
}

\begin{abstract}
This paper describes the EMG30 mechanical and electrical modeling and its simulation resorting to SimTwo (Robot@Factory mobile robot competition official simulator). It is described the developed setup applied to obtain the experimental data that was used to estimate the actuator parameters. It was obtained an electro-mechanical dynamical model that describes the motor, its gear box, and the encoder. The motivation to model and simulate the EMG30 is the fact that it is an actuator worldwide popular in the mobile robotics domain, being a low cost 12v motor equipped with encoders and a 30:1 reduction gearbox. The Goal of this work is to provide more realism and new features to the Robot@Factory official simulator, allowing participating teams to produce and validate different robot prototypes and its software, reducing considerably the development time.
\end{abstract}

\section{Introduction}

Robotic competitions are an excellent way to foster research and to attract students to technological areas [1]. The robotic competitions present standard problems that can be used as a benchmark, in order to evaluate and to compare the performances of different approaches. Although there are many robotic competitions [2-5], there is the need to create new ones, in order to solve new challenges. The factory environment is a prime candidate to use robots in a variety of tasks. A competition where mobile robots are tackling transportation problems in the shop floor is a

\footnotetext{
J. Gonçalves $(\bowtie) \cdot J$. Lima

INESC TEC (Formerly INESC Porto) and Polytechnic Institute of Bragança,

Bragança, Portugal

P. J. Costa - A. P. Moreira

INESC TEC (Formerly INESC Porto) and Faculty of Engineering, University of Porto,

Porto, Portugal
} 
challenge that can foster new advances in service robots and manufacturing [6,7]. The Robot@Factory competition presents problems that occur when using mobile robots to perform transportation tasks. The robots must be able to navigate, cooperate, and to self-localize in an emulated factory plant, to transport and handle materials in an efficient way [8].

This paper describes the EMG30 mechanical and electrical modeling and its simulation resorting to SimTwo. SimTwo is a realistic simulation software that can support several types of robots. Its main purpose is the simulation of mobile robots that can have wheels or legs, although industrial robots, conveyor belts, and lighter-than-air vehicles can also be defined. Basically any type of terrestrial robot definable with rotative joints and/or wheels can be simulated in this software [9].

The motivation to model and simulate the EMG30 is the fact that it is an actuator worldwide popular in the mobile robotics domain, being a low $\operatorname{cost} 12 \mathrm{v}$ motor equipped with encoders and a 30:1 reduction gearbox, and also to provide more realism and new features to SimTwo (the Robot@ Factory official simulator), allowing participating teams to produce and validate different robot prototypes and its software, reducing considerably the development time. The paper is organized as follows: After a brief introduction it is described the developed setup applied to obtain the experimental data and the actuator parameters estimation. Then its simulation resorting to SimTwo is presented. Finally some conclusions and future work are presented.

\section{Modeling of the EMG30 Actuator}

The EMG30 is an actuator worldwide popular in the mobile robotics domain, being a low cost $12 \mathrm{v}$ motor equipped with encoders and a 30:1 reduction gearbox. The fact that it is equipped with encoders is an important feature because it provides important data to obtain the closed loop velocity control and to obtain relative measurements based on the odometry calculation [10]. An EMG30 is shown in Fig. 1.

The EMG30 model can be defined by the following equation, where $U_{a}$ is the converter output, $R_{a}$ is the equivalent resistor, $L_{a}$ is the equivalent inductance, $e$ is the back emf (electromotive force) voltage, $I_{a}$ is the motor current as expressed by Eq. (1).

$$
U_{a}=e+R_{a} i_{a}+L_{a} \frac{d i_{a}}{d t}
$$

The motor can provide a torque $T_{L}$ that will be applied to the load, being the developed torque $T_{d}$ subtracted by the friction torque, which is the sum of the static friction $T_{c}$ and viscous friction, as shown in Eq. (2).

$$
T_{L}=T_{d}-T_{c}-B \omega
$$


Fig. 1 EMG30 geared motor

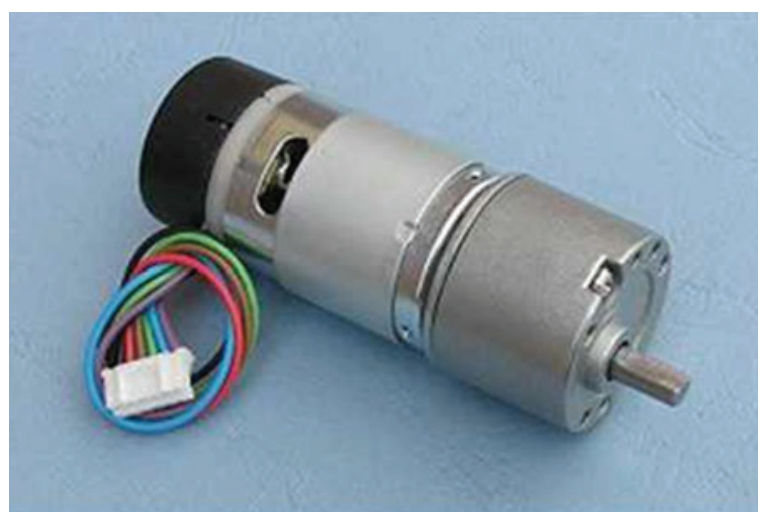

Current $i_{a}$ can be correlated with the developed torque $T_{d}$ through Eq. (3), the back emf voltage can be correlated with angular velocity through Eq. (4) and the load torque $T_{L}$ can be correlated with the moment of inertia and the angular acceleration through Eq. (5) [11].

$$
\begin{gathered}
T_{d}=K_{s} i_{a} \\
e=K_{s} \omega \\
T_{L}=J \dot{\omega}
\end{gathered}
$$

In order to obtain experimental data, a setup, shown in Fig. 2, was implemented. The experimental setup is based on the Arduino micro-controller, the L6207 Drive, a DC Power source, an EMG30 actuator, and the motor Load. The obtained data is the load angular velocity, the input voltage, and the motor current. Two tests were performed, the first was to obtain the step response for a $12 \mathrm{~V}$ input (transitory response data) and the second test was the steady state response for

Fig. 2 Experimental setup

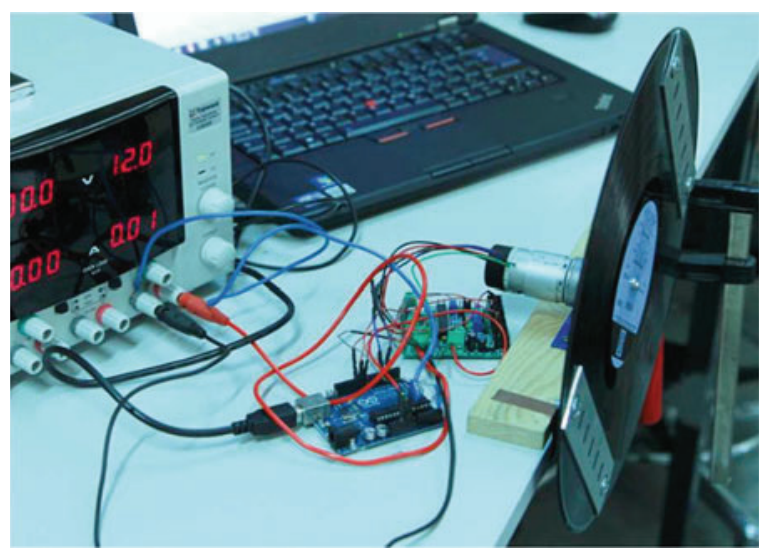


several input voltages (steady state data). Resorting to Eqs. (2), (3), and (5), Eq. (6) was obtained.

$$
\dot{\omega}=\frac{K_{s} i_{a}-T_{c}-B \omega}{J}
$$

After discretizing Eq. (6), Eq. (7) was obtained, where $\Delta \mathrm{T}$ is the sampling time (50 ms).

$$
\omega[k]=\omega[k-1]+\Delta T \frac{K_{S} i_{a}[k-1]-T_{c}-B \omega[k-1]}{J}
$$

By minimizing the sum of the absolute error between the estimated (7) and the real transitory response data (assuming initial know values for $R_{a}$ and $K_{s}$, parameters B and J were estimated. Then using Eqs. (1), (2), (3), (4), and (5) and assuming that voltage drop due to $L_{a}$ is negligible, Eq. (8) is obtained.

$$
J \dot{\omega}=\frac{K_{s}}{R_{a}}\left(U_{a}-K_{s} \omega\right)-B \omega-T_{c}
$$

Solving the first order differential equation, Eq. (9) is obtained:

$$
\begin{gathered}
\omega(t)=\frac{a}{b}\left(1-e^{-b t}\right) \\
a=\frac{K_{s} U_{a}-R_{a} T_{c}}{R_{a} J}
\end{gathered}
$$

where:

$$
b=\frac{k_{s}^{2}+R_{a} B}{R_{a} J}
$$

In steady state $\omega=a / b$, resulting in Eq. (12).

$$
\omega=\frac{K_{s}}{K_{s}^{2}+R_{a} B} U_{a}-\frac{R_{a} T_{c}}{K_{s}^{2}+R_{a} B}
$$

By minimizing the absolute error between estimated and the steady state data, assuming an initial value for $R_{a}$, parameters $K_{s}$ and $T_{c}$ are estimated. Finally resorting to Eq. (9), by minimizing the absolute error between the estimated data and the transitory response data, $R_{a}$ is estimated. The described optimization process must be repeated until the estimated parameters converge to their true values. Parameters such as $T_{c}, R_{a}$ and $k_{s}$ that are initially assumed as known are replaced by the estimated ones, every time the estimate process is repeated. The estimated and the real transitory and steady state responses are shown in Fig. 3.

The load has a known moment of inertia, given by the sum of three moments of inertia. A moment of inertia of a vinyl record $J_{d}=0.5 m_{d} r_{d}^{2}$ (where $m_{d}$ is the record mass and $r_{d}$ is its radius) summed with the moments of inertia of two planar rectangles, each one given by the equation $\mathrm{Jr}=\mathrm{m}\left(\mathrm{a}^{2}+\mathrm{b}^{2}\right) / 12$ (where $\mathrm{a}$ and $\mathrm{b}$ are 
(a)

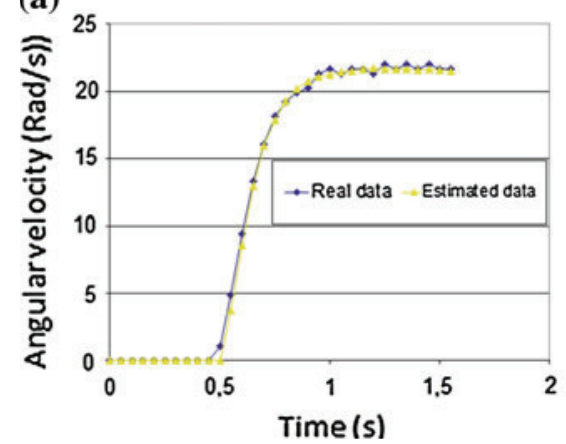

(b)

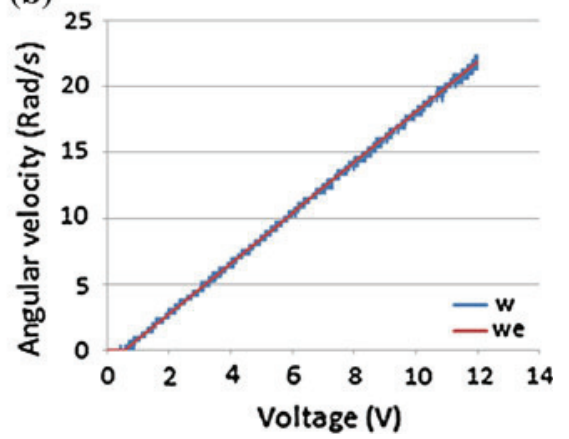

Fig. 3 a Motor transitory response. b Motor steady state response data

Table 1 EMG30 estimated parameters

\begin{tabular}{ll}
\hline Parameters & Value \\
\hline$k_{s}$ & 0.509 \\
$L_{a}$ & $3.4 \mathrm{E}-3$ \\
$R_{a}$ & 7.101 \\
$\mathrm{~B}$ & 0.000931 \\
$T_{c}$ & 0.0400 \\
$\mathrm{~J}$ & 0.00567 \\
$J_{M}$ & 0.00377 \\
$J_{L}$ & 0.0019 \\
\hline
\end{tabular}

the planar rectangles sides dimensions and $\mathrm{m}$ is the planar rectangles mass). Having in mind that the Parallel axis theorem has to be used in order to calculate the moment of inertia of the planar rectangles, $m r_{p}^{2}$ has to be summed to the previous calculated moment of inertia (where $\mathrm{m}$ is the rectangular plane mass and $r_{p}$ is the perpendicular distance between the axis of rotation and the axis that would pass through the Center of mass of each rectangular plane) [12]. In order to estimate the motor moment of inertia it is subtracted to the estimated value $\mathbf{J}$ the calculated moment of inertia, being $J_{L}$ the load moment of inertia and $J_{M}$ the moment of inertia. The estimated parameters are shown in Table 1, where the presented equivalent inductance was directly measured.

\section{Simulation of the EMG30 Resorting to SimTwo}

SimTwo is the official simulator of the Robot@Factory competition. The competition arena, shown in Fig. 4, emulates a factory shop floor where there are warehouses and machinery. A real robot prototyped with the EMG30 actuator moving in the competition arena is shown in Fig. 5. 
Fig. 4 Competition arena modeled in the SimTwo

Fig. 5 Robot in the competition arena

Fig. 6 EMG30 simulated in SimTwo
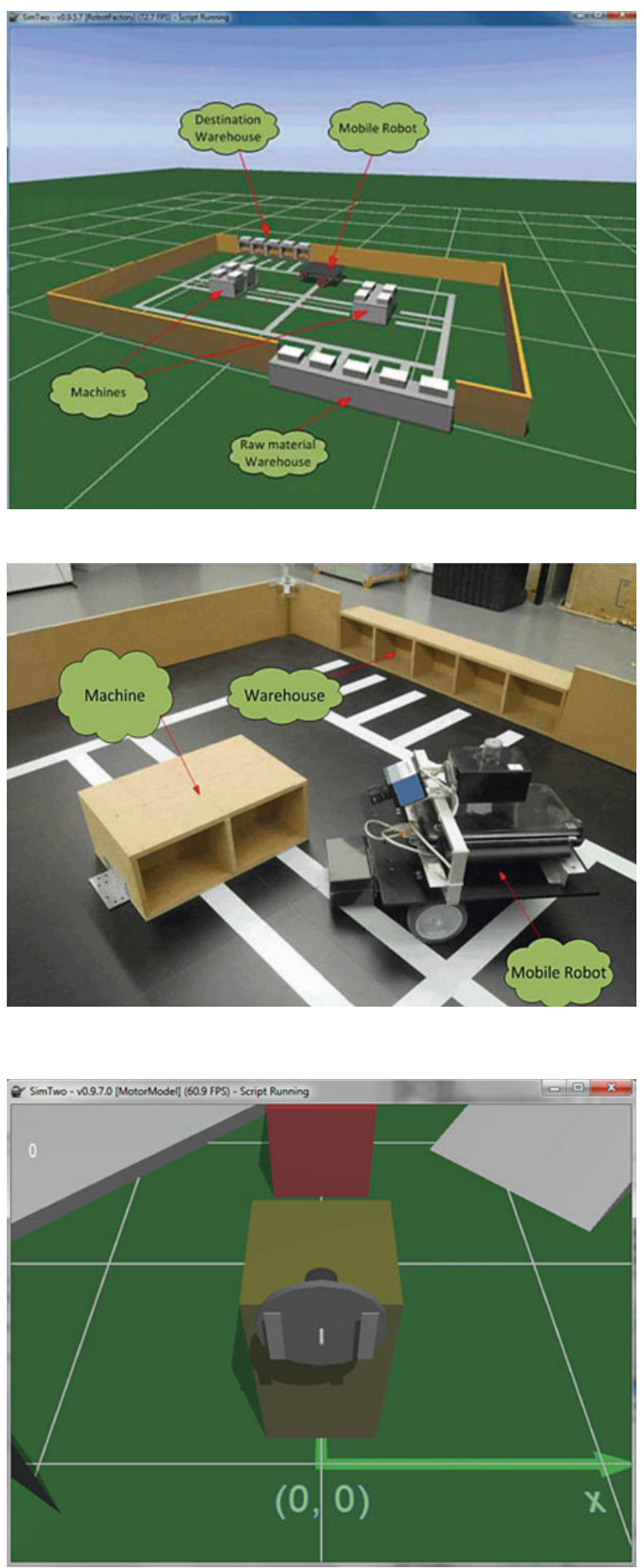
(a)

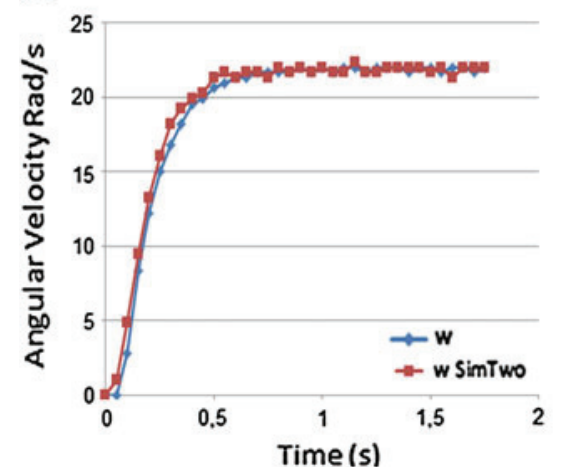

(b)

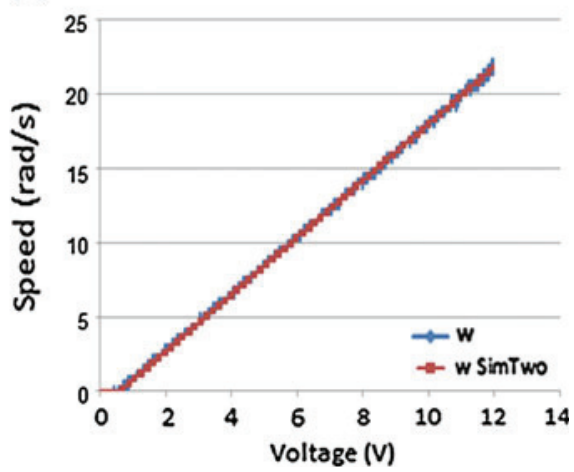

Fig. 7 a Actuator transitory response. b Actuator steady state response

In this section it is presented an example of a simulation of the EMG30 motor, being a very popular actuator among teams participating in the Robot@Factory competition. The experimental setup presented in Sect. 2 was simulated resorting to SimTwo, a snapshot of its simulation is shown in Fig. 6. The presented previously experiments were simulated, the real results and the simulated are shown in Fig. 7.

\section{Conclusions}

In this paper it is presented the EMG30 mechanical and electrical modeling and its simulation resorting to SimTwo (Robot@Factory mobile robot competition official simulator). It is described the developed setup applied to obtain the experimental data, that was used to estimate the actuator parameters. It was obtained an electromechanical dynamical model that describes the motor, its gear box, and the encoder. The motivation to model and simulate the EMG30 was the fact that it is an actuator worldwide popular in the mobile robotics domain, and in particular in the Robot@Factory participating teams, being a low cost $12 \mathrm{v}$ motor equipped with encoders and a 30:1 reduction gearbox. The referred robot competition can play an important role in education due to the inherent multi-disciplinary concepts that are involved, motivating students to technological areas. It also plays an important role in research and development, because it is expected that the outcomes that will emerge here, will later be transferred to other application areas, such as service robots and manufacturing. As future work the authors intend to produce robot code resorting to SimTwo with a robot prototyped with EMG30 actuators and apply it to the real robot.

Acknowledgments This work is partly financed by the ERDF-European Regional Development Fund through the COMPETE Programme (operational program for competitiveness) and by 
National Funds through the FCT-Fundação para a Ciência e a Tecnologia (Portuguese Foundation for Science and Technology) within project «FCOMP-01-0124-FEDER-022701».

\section{References}

1. Almeida L, Azevedo J, Cardeira C, Costa P, Fonseca P, Lima P, Ribeiro F, Santos V (2000) Fostering advances in research, development and education in robotics. In: Proceedings of the 4th Portuguese conference in automatic control

2. Browning B, Bruce J, Bowling M, Veloso M (2005) Ustp: Skills, tactics and plays for multirobot control in adversarial environments. IEEE J Control Syst Eng

3. Lund H, Pagliarinis L (2000) Robocup jr. with lego mindstorms. International conference on robotics and automation, San Francisco, CA, IEEE

4. Nakanishi R, Bruce J, Murakami K, Naruse T, Veloso M (2006) Cooperative 3-robot passing and shooting in the RoboCup small size league. In: Proceedings of the RoboCup symposium, Bremen, Germany

5. Ribeiro F, Moutinho I, Silva P, Fraga C, Pereira N (2004) Controlling omni-directional wheels of a RoboCup msl autonomous mobile robot. In Scientific Meeting of the Portuguese Robotics Open

6. Yuta S, Asama H, Thrun S, Prassler E, Tsubouchi T (2003) Field and service robotics, recent advances in research and applications. Volume 24 of Springer tracts in advanced robotics, Lake Yamanaka, Japan, 14-16 July 2003

7. Nambiar AN (2010) Challenges in sustainable manufacturing. In: Proceedings of the 2010 international conference on industrial engineering and operations management, Dhaka, Bangladesh, January 9-10 2010

8. Gonçalves J, Costa P, Lima J, Moreira A (2012) Manufacturing education and training resorting to a new mobile robot competition. Flexible Automation Intelligent Manufacturing (FAIM), Ferry Cruise Conference Helsinki-Stockholm-Helsinki, 10-13 June 2012

9. Costa P, Gonçalves J, Lima J, Malheiros P (2011) SimTwo realistic simulator: a tool for the development and validation of robot software. Int J Theory Appl Math Comput Sci

10. Borenstein J, Everett H, Feng J (1996) 'Where am I?' Sensors and methods for mobile robot positioning. Technical Report, The University of Michigan

11. Bishop R (2002) The mechatronics handbook. CRC Press, New York

12. Ramsey A (2009) "Dynamics", Cambridge Library Collection-Mathematics 